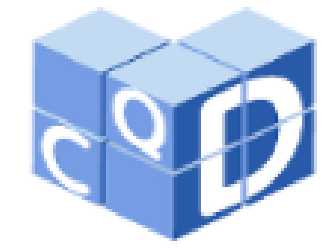

Revista Eletrônica

Paulista de Matemática

ISSN 2316-9664

Volume 14, fev. 2019

Edição Ermac

Luiz Victor Lima Macêdo Instituto Federal de Educação, Ciência e Tecnologia da Bahia victor.mat.ifba@gmail.com

Igor Breda Ferraço

Instituto Federal de Educação, Ciência e Tecnologia da Bahia igor.ferraco@ifba.edu.br

\section{Utilização da matriz de Leslie para estudar o crescimento populacional feminino da cidade de Eunápolis}

Using the Leslie Matrix to study female population growth in the city of Eunapolis

\section{Resumo}

Quando a variação de uma população se realiza em função do tempo, obtém-se um processo contínuo e discreto intitulado dinâmica de populações. Este processo é de fundamental importância no contexto das ciências aplicadas. O objetivo da dinâmica de populações é estudar as variações numéricas que sofrem as populações, determinar suas causas, preceder seu comportamento e analisar suas causas ecológicas. Neste trabalho, utilizamos o modelo matricial de Leslie para estudar o crescimento (ou decrescimento) populacional da cidade de Eunápolis, Bahia, a partir dos anos 2000, com o objetivo de comparar os resultados obtidos com os dados reais do sistema DATASUS. Para isto, utilizamos os dados contidos no mesmo para determinação dos parâmetros demográficos do modelo. Em seguida, realizamos simulações com o objetivo de estimar as populações nos anos de 2009 e 2018. Devido à proximidade dos dados reais com os resultados obtidos no ano de 2009 (não havia dados em relação a anos posteriores a 2012), concluímos que o modelo matricial de Leslie é uma ferramenta eficaz para realizar estimativas de populações femininas.

Palavras-Chave: Crescimento Populacional. Matriz de Leslie.

Abstract
When the variation of a population is performed as a func-
tion of time, a continuous and discrete process, called pop-
ulation dynamics, is obtained. The purpose of population
dynamics is to study the numerical variations suffered by
populations, determine their causes, precede their behavior
and analyze their ecological causes. In this paper, we use
the Leslie matrix model to study the growth (or decline) of
the city of Eunápolis, from the year 2000 , with the objective
of comparing the results obtained with the actual data from
the DATASUS system. For this, we use the data contained
therein to determine the demographic parameters of the
model. Then, we perform simulations in order to estimate
the populations in the years 2009 and 2018 . Due to the prox-
imity of the actual data with the results obtained in the year
2009 and the growth of the city, we conclude that the Leslie
matrix model is an effective tool to perform estimates of
female populations.

Keywords: Population Growth. Matrix of Leslie. 


\section{Introdução}

O crescimento ou decrescimento populacional é, sem dúvida, um fenômeno de estudo muito importante no contexto das ciências aplicadas. Neste sentido, a contribuição da Matemática está em fornecer modelos que permitam analisá-lo quantitativamente em função do tempo. Assim, diversos modelos matemáticos já foram elaborados com o objetivo de estudar este fenômeno, conforme pode ser visto em Anton e Rorres (2001), Bacaer (2011), Bassanezi (2013) e Sodré (2007).

O modelo matricial (ou matriz) de Leslie é assim denominado em homenagem ao seu criador, o fisiólogo Patrick Holt Leslie (1900-1972). Leslie, conforme Bacaër (2011), nasceu em 1900 na Escócia e obteve o título de Bacharel em Fisiologia em 1921. Contudo, foi impedido de terminar seus estudos médicos devido a problemas de saúde. Assim, trabalhou por alguns anos no Departamento de Bacteriologia, até que em 1935 se interessou por Estatística e se juntou ao centro de pesquisa Bureau of Animal Population, que tinha como objetivo estudar as flutuações de populações animais. Mais tarde, em 1945, publicou seu artigo mais famoso, intitulado On the use of matrices in certain populations mathematics, onde apresenta o modelo aqui utilizado.

Neste trabalho, utilizamos o modelo matricial de Leslie para estudar o crescimento ou decrescimento populacional feminino da cidade de Eunápolis, a partir dos anos 2000, com o objetivo de estimar a população em anos posteriores e comparar com os dados reais obtidos no sistema DATASUS. Assim, pretendemos verificar a aplicabilidade do modelo, tendo em vista as mudanças e o crescimento que a cidade sofreu desde que foi emancipada em 1988. A escolha do tempo inicial (ano 2000) para este estudo justifica-se pela melhor adequação dos dados do sistema ao modelo.

Este artigo possui a seguinte estrutura: de início, na seção 2, apresentaremos o modelo matricial de Leslie. Detalhes adicionais poderão ser vistos em Anton e Rorres (2001) e Bacaer (2011). Em seguida, na seção 3, apresentaremos os dados do sistema DATASUS, a metodologia aplicada e discutiremos os resultados. Por fim, na seção 4, apresentaremos as conclusões.

\section{Modelo matricial de Leslie}

Suponhamos que seja $I$ a idade máxima atingida pelas fêmeas de uma determinada população. Ao dividirmos a população em $n$ faixas etárias, temos que a duração de cada faixa etária é dada por $\frac{I}{n}$, conforme a Tabela 1.

Tabela 1 - Faixas Etárias

\begin{tabular}{|c|c|}
\hline Faixa Etária & Intervalo de Idade \\
\hline 1 & {$[0, I / n[$} \\
\hline 2 & {$[I / n, 2 I / n[$} \\
\hline 3 & {$[2 I / n, 3 I / n[$} \\
\hline$\vdots$ & $\vdots$ \\
\hline$n-1$ & {$[(n-2) I / n,(n-1) I / n]$} \\
\hline$n$ & {$[(n-1) I / n, I]$} \\
\hline
\end{tabular}


Suponhamos que seja conhecido o número de fêmeas em cada uma das $n$ faixas etárias no instante $t=0$. Em particular, $x_{1}^{(0)}$ na primeira faixa, $x_{2}^{(0)}$ na segunda faixa, e assim por diante. Com estes $n$ números, formaremos o vetor-coluna

$$
x^{(0)}=\left[\begin{array}{c}
x_{1}^{(0)} \\
x_{2}^{(0)} \\
\vdots \\
x_{n}^{(0)}
\end{array}\right]
$$

que chamaremos de vetor de distribuição etária inicial.

Conforme o tempo avança, o número de fêmeas em cada uma das $n$ faixas etárias muda por conta de três processos biológicos: nascimento, morte ou envelhecimento. Descrevendo estes processos quantitativamente, poderemos projetar o vetor de distribuição inicial para o futuro.

A maneira mais fácil de estudar o processo de envelhecimento é observar a população em intervalos discretos de tempo, ou seja, $t_{0}, t_{1}, t_{2}, \ldots, t_{k}$. Desta forma, para observamos a variação da população em cada faixa devido ao envelhecimento, façamos:

$$
\begin{gathered}
t_{0}=0 \\
t_{1}=I / n \\
t_{2}=2 I / n \\
\vdots \\
t_{k}=\mathrm{kI} / \mathrm{n}
\end{gathered}
$$

Daí, todas as fêmeas na $(i+1)$ - ésima faixa etária no instante $t_{k+1}$, estavam na $i-$ ésima faixa etária no instante $t_{k}$.

Os processos de nascimento e morte entre dois tempos de observação sucessivos podem ser estudados de acordo com os parâmetros demográficos expostos na Tabela 2 a seguir.

Tabela 2 - Parâmetros demográficos

\begin{tabular}{|c|l|}
\hline$a_{i}$ & $\begin{array}{l}\text { Número médio de filhas nascidas por } \\
\text { fêmea durante o tempo em que ela está } \\
\text { na } i \text { - ésima faixa etária. }\end{array}$ \\
\hline$(i=1,2,3, \ldots, n)$ & $\begin{array}{l}\text { Fração de fêmeas na } i \text { - ésima etária } \\
\text { que se espera que vá sobreviver e pas- } \\
\text { sar para a }(i+1)-\text { ésima faixa etá- } \\
\text { ria. }\end{array}$ \\
\hline
\end{tabular}


De acordo com as definições, temos que: $a_{i} \geq 0$, para $i=1,2,3, \ldots, n$ e $0<b_{i} \leq 1$, para $i=1,2,3, \ldots, n-1$. Suporemos ainda que: nenhum dos $b_{i}$ é nulo, pois desta maneira nenhuma fêmea sobreviveria a partir da $i$ - ésima faixa etária; pelo menos um dos $a_{i}$ deve ser positivo, de modo que haja algum nascimento.

Assim, o vetor de distribuição etária no instante $t_{k}$ é definido por

$$
x^{(k)}=\left[\begin{array}{c}
x_{1}^{(k)} \\
x_{2}^{(k)} \\
\vdots \\
x_{n}^{(k)}
\end{array}\right]
$$

em que $x_{i}^{(k)}$ é o número de fêmeas na $i$ - ésima faixa etária no instante $t_{k}$.

Desta forma, no instante $t_{k}$, as fêmeas na primeira faixa etária são exatamente as filhas nascidas entre os instantes $t_{k-1}$ e $t_{k}$. Poderíamos escrever

$$
\left\{\begin{array}{c}
\text { número } \\
\text { de } \\
\text { fêmeas } \\
\text { na faixa } \\
\text { etária } \\
1 \\
\text { no tempo } t_{k}
\end{array}\right\}=\left\{\begin{array}{c}
\text { número de } \\
\text { filhas nascidas } \\
\text { das fêmeas na } \\
\text { faixa etária } 1 \\
\text { entre os tempos } \\
t_{k-1} \text { e } t_{k}
\end{array}\right\}+\left\{\begin{array}{c}
\text { número de filhas } \\
\text { nascidas das fêmeas } \\
\text { na faixa etária } 2 \\
\text { entre os tempos } \\
t_{k-1} \text { e } t_{k}
\end{array}\right\}+\cdots+\left\{\begin{array}{c}
\text { número de } \\
\text { filhas nascidas } \\
\text { das fêmeas na } \\
\text { faixa etárian } \\
\text { entre os tempos } \\
t_{k} \text { e } t_{k-1}
\end{array}\right\}
$$

ou, matematicamente,

$$
x_{1}^{(k)}=a_{1} x_{1}^{(k-1)}+a_{2} x^{(k-1)}+\cdots+a_{n} x_{n}^{(k-1)}
$$

As fêmeas na $(i+1)$ - ésima faixa etária $(i=1,2,3, \ldots, n-1)$ no instante $t_{k}$ são aquelas que estavam na $i$ - ésima faixa etária no instante $t_{k-1}$ e que ainda vivem no instante $t_{k}$. Assim,

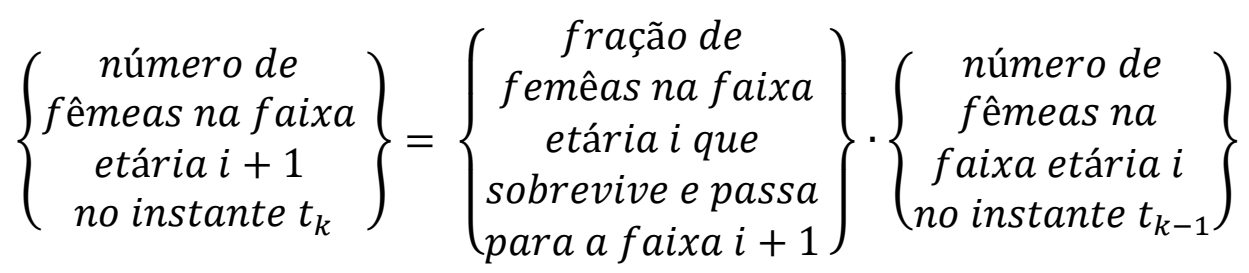

ou, matematicamente,

$$
x_{i+1}^{(k)}=b_{i} \cdot x_{i}^{(k-1)}, i=1,2, \ldots, n-1
$$

Utilizando notação matricial, podemos escrever as equações (4) e (5) da seguinte maneira: 


$$
\left[\begin{array}{c}
x_{1}^{(k)} \\
x_{2}^{(k)} \\
x_{3}^{(k)} \\
\vdots \\
x_{n}^{(k)}
\end{array}\right]=\left[\begin{array}{ccccc}
a_{1} & a_{2} & a_{3} & \cdots & a_{n} \\
b_{1} & 0 & 0 & \cdots & 0 \\
0 & b_{2} & 0 & \cdots & 0 \\
\vdots & 0 & \ddots & 0 & 0 \\
0 & 0 & 0 & b_{n} & 0
\end{array}\right]\left[\begin{array}{c}
x_{1}^{(k-1)} \\
x_{2}^{(k-1)} \\
x_{3}^{(k-1)} \\
\vdots \\
x_{n}^{(k-1)}
\end{array}\right]
$$

ou, simplificadamente,

$$
x^{(k)}=L \cdot x^{(k-1)}, \quad k=1,2, \ldots
$$

em que $L$ é a matriz de Leslie

$$
L=\left[\begin{array}{ccccc}
a_{1} & a_{2} & a_{3} & \cdots & a_{n} \\
b_{1} & 0 & 0 & \cdots & 0 \\
0 & b_{2} & 0 & \cdots & 0 \\
\vdots & 0 & \ddots & 0 & 0 \\
0 & 0 & 0 & b_{n} & 0
\end{array}\right]
$$

Observe que de (7), obtemos:

$$
\begin{gathered}
x^{(1)}=L \cdot x^{(0)} \\
x^{(2)}=L \cdot x^{(1)}=L^{2} \cdot x^{(0)} \\
x^{(3)}=L \cdot x^{(2)}=L^{3} \cdot x^{(0)} \\
x^{(k)}=L \cdot x^{(k-1)}=L^{k} \cdot x^{(0)}
\end{gathered}
$$

Portanto, ao conhecermos a distribuição etária inicial $x^{(0)}$ e a matriz de Leslie $L$, poderemos determinar a distribuição etária das fêmeas em tempos posteriores.

\section{Aplicação do modelo na cidade de Eunápolis}

Para estudarmos o crescimento ou decrescimento populacional feminino da cidade de Eunápolis, fizemos $I=100, n=10$ e escolhemos $t_{0}=2000$. Assim, a duração da faixa etária foi de 10 anos de acordo com os intervalos [0 - 9], [10 - 19], [20 - 29], ... , [90 - 99].

Em relação à população feminina residente na cidade de Eunápolis ano 2000, obtivemos os seguintes dados através do sistema, conforme consta na Figura 1.

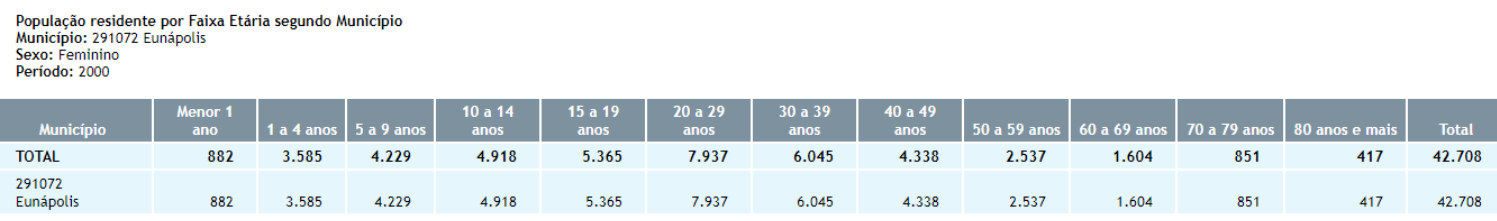

Figura 1 - População residente na cidade de Eunápolis no ano 2000

Fonte: http://tabnet.datasus.gov.br/cgi/tabcgi.exe?ibge/cnv/popba.def 
Com isto, o vetor de distribuição inicial $x^{(0)}$ é dado por:

$$
x^{(0)}=\left[\begin{array}{c}
8696 \\
10283 \\
7937 \\
6045 \\
4338 \\
2537 \\
1604 \\
851 \\
417 \\
0
\end{array}\right] .
$$

Cabe ressaltar que por não haver os valores exatos em relação às faixas etárias [80 - 89], [90 - 99], supomos que as 417 mulheres pertenciam à penúltima.

Conhecido então a distribuição etária inicial, faltava-se determinar os parâmetros de nascimento e sobrevivência para compormos a matriz de Leslie.

Para determinação dos parâmetros de natalidade $a_{i}$, com $i=1,2, \ldots, n$, utilizamos as informações contidas no sistema DATASUS, conforme as Figuras 2 e 3. Na Figura 2 temos o número de filhas nascidas por residência divididas em faixas etárias, enquanto que na Figura 3 por ocorrência.

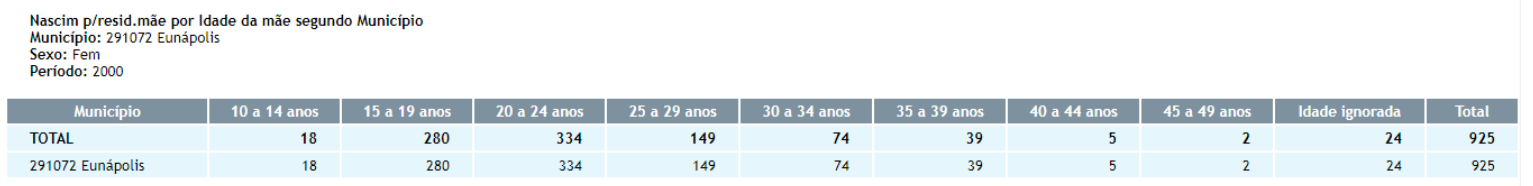

Figura 2 - Mulheres nascidas por residência por faixa etária no ano 2000

Fonte: http://tabnet.datasus.gov.br/cgi/tabcgi.exe?sinasc/cnv/nvba.def

\begin{tabular}{|l|r|r|r|r|r|r|r|r|r|r|r|r|}
\hline \multicolumn{1}{|c|}{ Municipio } & 10 a 14 anos & 15 a 19 anos & 20 a 24 anos & 25 a 29 anos & 30 a 34 anos & 35 a 39 anos & 40 a 44 anos & 45 a 49 anos & Idade ignorada & Total \\
\hline TOTAL & 20 & 379 & 421 & 188 & 98 & 53 & 12 & 25 & 1.208 \\
\hline 291072 Eunápolis & 20 & 379 & 421 & 188 & 98 & 53 & 12 & 2 & 35 & 1.208 \\
\hline
\end{tabular}

Figura 3 - Mulheres nascidas por ocorrência por faixa etária no ano de 2000

Fonte: http://tabnet.datasus.gov.br/cgi/tabcgi.exe?sinasc/cnv/nvba.def

Em resumo, o número de filhas nascidas em Eunápolis no ano 2000 pode ser visto na Tabela 3. Diferente do que foi feito em Macedo e Ferraço (2018), as 59 filhas nascidas que tiveram a idade da mãe ignorada, não foram adicionadas.

Tabela 3 - Número de filhas nascidas vivas na cidade de Eunápolis no ano 2000

\begin{tabular}{|c|c|}
\hline Faixa Etária & Filhas Nascidas \\
\hline$[00-09]$ & 0 \\
\hline$[10-19]$ & 697 \\
\hline$[20-29]$ & 1092 \\
\hline
\end{tabular}




\begin{tabular}{|c|c|}
\hline$[30-39]$ & 264 \\
\hline$[40-49]$ & 21 \\
\hline$[50-59]$ & 0 \\
\hline$[60-69]$ & 0 \\
\hline$[70-79]$ & 0 \\
\hline$[80-89]$ & 0 \\
\hline$[90-99]$ & 0 \\
\hline
\end{tabular}

Daí, determinamos os $a_{i}$ do seguinte modo:

$$
a_{i}=\frac{p_{i}}{\sum_{i}^{n} p_{i}}, i=1,2,3, \ldots, n
$$

em que $p_{i}$ representa o número de filhas na $i$ - ésima faixa etária.

Desta forma, os valores obtidos para os parâmetros de natalidade em cada faixa etária podem ser vistos na Tabela 4 a seguir.

Tabela 4 - Parâmetros de natalidade por faixa etária

\begin{tabular}{|c|c|}
\hline Faixa Etária & $a_{i}$ \\
\hline$[00-09]$ & 0 \\
\hline$[10-19]$ & 0,336 \\
\hline$[20-29]$ & 0,526 \\
\hline$[30-39]$ & 0,127 \\
\hline$[40-49]$ & 0,01 \\
\hline$[50-59]$ & 0 \\
\hline$[60-69]$ & 0 \\
\hline$[70-79]$ & 0 \\
\hline$[80-89]$ & 0 \\
\hline$[90-99]$ & 0 \\
\hline
\end{tabular}

Em seguida, calculamos as taxas de sobrevivência, isto é, os $b_{i}, i=1,2,3, \ldots, n-1$. Para isto, também utilizamos as informações contidas no sistema DATASUS, conforme Figuras 4 e 5.

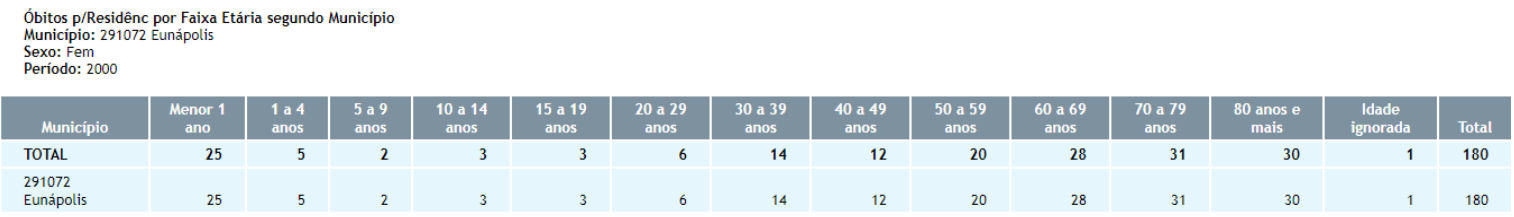

Figura 4 - Número de óbitos por residência por faixa etária Fonte: http://tabnet.datasus.gov.br/cgi/tabcgi.exe?sim/cnv/obt10ba.def

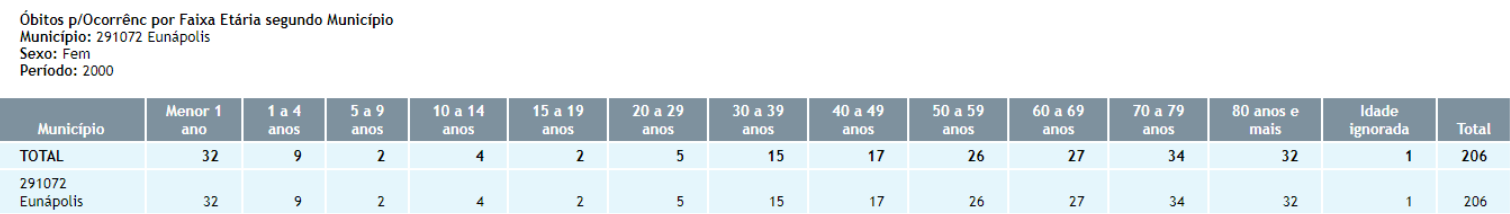

Figura 5 - Número de óbitos por ocorrência por faixa etária

Fonte: http://tabnet.datasus.gov.br/cgi/tabcgi.exe?sim/cnv/obt10ba.def

MACÊDO, L. V. L.; FERRAÇO, I. B. Utilização da matriz de Leslie para estudar o crescimento populacional feminino da cidade de Eunápolis. C.Q.D.- Revista Eletrônica Paulista de Matemática, Bauru, v. 14, p. 110-121, fev. 2019. Edição Ermac.

DOI: 10.21167/cqdvol14ermac201923169664lvlmibf110121 Disponível em: https://www.fc.unesp.br/\#!/departamentos/matematica/revista-cqd/ 
Em resumo, o número de óbitos em Eunápolis no ano 2000 pode ser visto na Tabela 5. Os 2 óbitos que tiveram ignorada, não foram adicionados, diferente do que foi feito em Macedo e Ferraço (2018).

Tabela 5 - Número de óbitos femininos na cidade de Eunápolis no ano de 2000

\begin{tabular}{|c|c|}
\hline Faixa Etária & Óbitos \\
\hline$[00-09]$ & 75 \\
\hline$[10-19]$ & 12 \\
\hline$[20-29]$ & 11 \\
\hline$[30-39]$ & 29 \\
\hline$[40-49]$ & 29 \\
\hline$[50-59]$ & 46 \\
\hline$[60-69]$ & 55 \\
\hline$[70-79]$ & 65 \\
\hline$[80-89]$ & 62 \\
\hline$[90-99]$ & 0 \\
\hline
\end{tabular}

Observe que supomos os óbitos classificados por 80 anos ou mais como pertencentes à penúltima faixa etária.

A partir daí, determinamos os $b_{i}, i=1,2,3, \ldots, n-1$ do seguinte modo:

$$
b_{i}=\frac{s_{i}}{x_{i}}, i=1,2,3, \ldots, n-1
$$

em que $s_{i}$ representa o número de fêmeas sobreviventes na $i$ - ésima faixa etária.

Desta forma, os parâmetros de sobrevivência $b_{i}$ obtidos podem ser vistos na Tabela 6 a seguir.

Tabela 6 - Parâmetros de mortalidade por faixa etária

\begin{tabular}{|c|c|}
\hline Faixa Etária & $b_{i}$ \\
\hline$[00-09]$ & 0,991 \\
\hline$[10-19]$ & 0,998 \\
\hline$[20-29]$ & 0,998 \\
\hline$[30-39]$ & 0,995 \\
\hline$[40-49]$ & 0,993 \\
\hline$[50-59]$ & 0,981 \\
\hline$[60-69]$ & 0,965 \\
\hline$[70-79]$ & 0,923 \\
\hline$[80-89]$ & 0,851 \\
\hline$[90-99]$ & - \\
\hline
\end{tabular}

Substituindo os valores obtidos em (6), temos a seguinte equação matricial: 


$$
\left[\begin{array}{c}
x_{1}^{(1)} \\
x_{2}^{(1)} \\
x_{3}^{(1)} \\
x_{5}^{(1)} \\
x_{6}^{(1)} \\
x_{7}^{(1)} \\
x_{8}^{(1)} \\
x_{9}^{(1)} \\
x_{10}^{(1)}
\end{array}\right]=\left[\begin{array}{cccccccccc}
0 & 0,336 & 0,526 & 0,127 & 0,01 & 0 & 0 & 0 & 0 & 0 \\
0,991 & 0 & 0 & 0 & 0 & 0 & 0 & 0 & 0 & 0 \\
0 & 0,998 & 0 & 0 & 0 & 0 & 0 & 0 & 0 & 0 \\
0 & 0 & 0,998 & 0 & 0 & 0 & 0 & 0 & 0 & 0 \\
0 & 0 & 0 & 0,995 & 0 & 0 & 0 & 0 & 0 & 0 \\
0 & 0 & 0 & 0 & 0,993 & 0 & 0 & 0 & 0 & 0 \\
0 & 0 & 0 & 0 & 0 & 0,981 & 0 & 0 & 0 & 0 \\
0 & 0 & 0 & 0 & 0 & 0 & 0,965 & 0 & 0 & 0 \\
0 & 0 & 0 & 0 & 0 & 0 & 0 & 0,923 & 0 & 0 \\
0 & 0 & 0 & 0 & 0 & 0 & 0 & 0 & 0,851 & 0
\end{array}\right]\left[\begin{array}{c}
8696 \\
7937 \\
6045 \\
4338 \\
2537 \\
1604 \\
851 \\
417 \\
0
\end{array}\right]
$$

Daí,

$$
x^{(1)}=\left[\begin{array}{c}
8441 \\
8618 \\
10262 \\
7921 \\
6015 \\
4308 \\
2489 \\
1548 \\
785 \\
355
\end{array}\right] .
$$

Portanto, existem 8441 mulheres na primeira faixa etária, 8618 na segunda, e assim sucessivamente. Na Tabela 7, encontram-se os resultados obtidos pelo modelo em comparação com os dados reais do sistema DATASUS.

Tabela 7 - Comparação entre os resultados e os dados reais por faixa etária

\begin{tabular}{|c|c|c|}
\hline Faixa Etária & Resultados do Modelo & DATASUS \\
\hline$[0-9]$ & 8441 & 9923 \\
\hline$[10-19]$ & 8618 & 8938 \\
\hline$[20-29]$ & 10262 & 10166 \\
\hline$[30-39]$ & 7921 & 7723 \\
\hline$[40-49]$ & 6015 & 5909 \\
\hline$[50-59]$ & 4308 & 3886 \\
\hline$[60-69]$ & 2489 & 2163 \\
\hline$[70-79]$ & 1548 & 1225 \\
\hline$[80-89]$ & 785 & 589 \\
\hline$[90-99]$ & 355 & - \\
\hline
\end{tabular}

Em relação ao valor total, consideramos a proximidade satisfatória: 50522 nos dados reais e 50742 na simulação do modelo, ou seja, uma diferença de 220 mulheres.

Na Tabela 8 a seguir, consta a diferença entre os mesmos por faixa etária. 
Tabela 8 - Diferença entre os resultados e os dados reais no ano de 2009

\begin{tabular}{|c|c|}
\hline Faixa Etária & Diferença \\
\hline$[0-9]$ & 1482 \\
\hline$[10-19]$ & 320 \\
\hline$[20-29]$ & 96 \\
\hline$[30-39]$ & 198 \\
\hline$[40-49]$ & 106 \\
\hline$[50-59]$ & 422 \\
\hline$[60-69]$ & 323 \\
\hline$[70-79]$ & 326 \\
\hline$[80-89]$ & 196 \\
\hline$[90-99]$ & - \\
\hline
\end{tabular}

Note que ao calcularmos $x^{(2)}$, obteremos uma estimativa da população feminina da cidade de Eunápolis no ano de 2018. De (6), vem que

$$
\left[\begin{array}{l}
x_{1}^{(2)} \\
x_{2}^{(2)} \\
x_{3}^{(2)} \\
x_{5}^{(2)} \\
x_{6}^{(2)} \\
x_{7}^{(2)} \\
x_{8}^{(2)} \\
x_{9}^{(2)} \\
x_{10}^{(2)}
\end{array}\right]=\left[\begin{array}{cccccccccc}
0 & 0,336 & 0,526 & 0,127 & 0,01 & 0 & 0 & 0 & 0 & 0 \\
0,991 & 0 & 0 & 0 & 0 & 0 & 0 & 0 & 0 & 0 \\
0 & 0,998 & 0 & 0 & 0 & 0 & 0 & 0 & 0 & 0 \\
0 & 0 & 0,998 & 0 & 0 & 0 & 0 & 0 & 0 & 0 \\
0 & 0 & 0 & 0,995 & 0 & 0 & 0 & 0 & 0 & 0 \\
0 & 0 & 0 & 0 & 0,993 & 0 & 0 & 0 & 0 & 0 \\
0 & 0 & 0 & 0 & 0 & 0,981 & 0 & 0 & 0 & 0 \\
0 & 0 & 0 & 0 & 0 & 0 & 0,965 & 0 & 0 & 0 \\
0 & 0 & 0 & 0 & 0 & 0 & 0 & 0,923 & 0 & 0 \\
0 & 0 & 0 & 0 & 0 & 0 & 0 & 0 & 0,851 & 0
\end{array}\right]\left[\begin{array}{c}
8441 \\
8618 \\
10262 \\
7921 \\
6015 \\
4308 \\
2489 \\
1548 \\
785 \\
355
\end{array}\right]
$$

Daí,

$$
x^{(2)}=\left[\begin{array}{c}
9359 \\
8365 \\
8601 \\
10241 \\
7881 \\
5972 \\
4226 \\
2401 \\
1429 \\
668
\end{array}\right]
$$

Assim, existem 9359 na primeira faixa, 8365 na segunda e assim sucessivamente. No total, o modelo estimou que existem 59413 mulheres na cidade de Eunápolis. Contudo, não existem informações acerca da população feminina da cidade em 2018 no DATASUS. Assim, não houve como realizar comparações com os resultados obtidos. 


\section{Considerações finais}

Neste trabalho, utilizamos o modelo matricial de Leslie para estudar o crescimento (ou decrescimento) populacional feminino da cidade de Eunápolis a partir do ano 2000. Para isto, utilizamos os dados do sistema DATASUS com objetivo de comparar os resultados com os dados obtidos em anos posteriores no mesmo sistema.

No processo de determinação dos parâmetros demográficos (Tabela 2), percebemos a importância dos mesmos, pois é a partir deles que se terá toda a distribuição etária posterior, conforme visto em (7). Além disto, é essencial que os métodos utilizados para determiná-los sejam fiéis a população em estudo, pois empregá-los de maneira errada poderiam levar a conclusões errôneas sobre o crescimento (ou descrecimento) da população estudada.

Em relação ao ano de 2009, consideramos os resultados obtidos satisfatórios, devido à proximidade dos resultados com os dados reais do sistema, como visto na Tabela 7, 8 e 9. Mais ainda em relação ao valor total, conforme a Tabela 8. Como no sistema DATASUS não havia informações quanto à população residente após o ano de 2012, não foi possível comparar os resultados do ano de 2018 com dados reais.

Cabe ressaltar que, embora a equação (7) apresente a distribuição etária da população para qualquer instante de tempo, ela não fornece uma ideia geral da dinâmica de crescimento (ou decrescimento). Para isto, deve-se investigar os autovalores e autovetores da matriz de Leslie associada. Este estudo, no entanto, ficará para trabalhos futuros.

Por fim, concluímos que o modelo matricial de Leslie é uma ferramenta eficaz para realizar estimativas de populações femininas. 


\section{Referências}

ANTON, H. RORRES, C. Álgebra linear e aplicações. Trad: Claus Ivo Doering. 8. ed. Porto Alegre: Bookman, 2001.

BACAER, N. A Shorty history of mathematical populational dinamycs. Londres: Springer-Verlag, 2011.

BASSANEZI, C. B. Modelagem matemática. [2013]. Disponível em: <http://posmat.ufabc.edu.br/inverno/wp-content/uploads/2013/04/minicurso-ufabc1.pdf $>$. Acesso em: 29 nov. 2018.

MACEDO, L. V. L.; FERRAÇO, B. I. Utilização da Matriz de Leslie para estudar o crescimento populacional da cidade de Eunápolis - BA. In: ENCONTRO REGIONAL DE MATEMÁTICA APLICADA E COMPUTACIONAL, 5., 2018, Bauru. Caderno de trabalhos completos e resumos... Bauru: Unesp, Faculdade de Ciências, 2018, p.485-486. Disponível em: <https://drive.google.com/file/d/1-

8SZZnioKApdmQG6BaOTltJARzfwAB9P/view>. Acesso em: 29 nov. 2018.

SODRÉ, U. Modelos matemáticos. Londrina, 2007. Disponível em:

<http://www.uel.br/projetos/matessencial/superior/pdfs/modelos.pdf $>$. Acesso em: 29 nov. 2018.

TABNET DATASUS. População Residente - Bahia. [2018?] Disponível em:

$\langle$ http://tabnet.datasus.gov.br/cgi/deftohtm.exe?ibge/cnv/popba.def $>$. Acesso em: 10 ago. 2018.

TABNET DATASUS. Nascidos Vivos - Bahia. [2018?]. Disponível em: <http://tabnet.datasus.gov.br/cgi/deftohtm.exe?sinasc/cnv/nvuf.def>. Acesso em: 10 ago. 2018.

TABNET DATASUS. Mortalidade - Bahia. [2018?] Disponível em: <http://tabnet.datasus.gov.br/cgi/deftohtm.exe?sim/cnv/obt10uf.def >. Acesso em: 10 ago. 2018. 